\title{
Nature of extrinsic and intrinsic self-trapping of charge carriers in underdoped cuprate high- $T_{\mathrm{c}}$ superconductors
}

\author{
O.K. Ganiev \\ Department of Physics, National University of Uzbekistan, 100174 Tashkent, Uzbekistan
}

Received August 1, 2016, in final form September 17, 2016

Nature of extrinsic and intrinsic self-trapping (ST) of charge carriers in cuprates have been studied theoretically. The binding energies and radii of the extrinsic and intrinsic large polarons and bipolarons in cuprates are calculated variationally using the continuum model and adiabatic approximation. We have shown that the extrinsic and intrinsic three-dimensional (3D) large bipolarons exist in underdoped cuprates at $\eta=\varepsilon_{\infty} / \varepsilon_{0}<0.127$ and $\eta<0.138$, respectively [where $\varepsilon_{\infty}\left(\varepsilon_{0}\right)$ is the optic (static) dielectric constant].

Key words: polaron, bipolaron, self-trapping, high- $T_{C}$ superconductors

PACS: 71.38.-k, 74.72.- $h$

\section{Introduction}

It is well known that electron-phonon coupling (EPC) is one of the most common and fundamental interactions in solids. In particular, a strong EPC in high-temperature superconducting cuprates (HTSC) was reported by a lot of experiments, which implies that EPC plays an important role in pairing. Accordingly, charge carriers in polar materials interacting with the phonon field can cause the formation of self-trapped polarons and bipolarons. The interest to the polarons and bipolarons is caused by their important role in explaining many characteristics of HTSC compounds (see [1, 2] and references therein). The ground state of an undoped HTSC is an antiferromagnet (AF) whose doping by holes leads to the phase showing high temperatures of the superconducting (SC) transition. The extended $t-t^{\prime}-t^{\prime \prime}-J$-Hubbard model, where a hole in a two-dimensional (2D) AF is simultaneously magnetic and a lattice polaron that moves through the lattice emitting and absorbing magnons and phonons, is used in many papers for calculation of spectral properties of underdoped HTSC compounds [3]. According to the band calculations and angle resolved-photoemission spectroscopy (ARPES) data [4, 5], the electronic structure of the parent cuprate compounds is well described by a three-band Hubbard model and the oxygen valence band lies within the Mott-Hubbard gap. Furthermore, according to the combined study of the ARPES and quantum Monte Carlo simulations, there was proposed a novel polaronic metallic state in underdoped cuprates [3]. The variety of opinions range from a complete negation of the role of EPC in the physics of HTSC [6, 7] to the statement that the binding energy of the polaron is an order of magnitude larger than any characteristic energy of the magnetic subsystem, and, therefore, exactly the magnetic system is irrelevant [8, 9]. Both of the above radical statements have been criticized many times. On the other hand, the success of the extended $t$ - $J$-Hubbard model in describing the ARPES [3, 10] and optical spectra [11] does not make it possible to completely disregard the role of the magnetic subsystem. Correspondingly, there is an opinion that interactions with both magnetic and lattice subsystems are important [12]. Results indicating an important role of the EPC in HTSC compounds are presented, with emphasis on its implications for ARPES and optical conductivity [12].

Hole doping of the cuprates produces first quasi-free holes having the mass $m_{\mathrm{h}}$ in the oxygen valence band. The hole carriers are assumed to be within both a three-dimensional (3D) and a 2D deformable 
medium, the last one being $\mathrm{CuO}_{2}$ layers [13]. In reality, however, no systems can be purely 2D, and therefore, the layered cuprate compounds may be approximated as a 3D deformable medium. There is also a convincing experimental evidence that the consideration of cuprates as 3D systems may appear to be more appropriate (see [14-17]. The experimental results presented in [16] indeed confirm that the holedoped system $\mathrm{La}_{2-x} \mathrm{Sr}_{x} \mathrm{CuO}_{4}$ (LSCO) becomes less 2D in the strongly localized state. In polar materials, the hole carriers interacting both with lattice vibrations (i.e., acoustic and optical phonons) and with lattice defects (e.g., dopants or impurities), can easily be self-trapped near the defects and in a defect-free deformable lattice. Actually, the carrier localization in the cuprates is interrelated and the quantitative theory of this phenomenon is still lacking. Particularly, possible roles of large- and small-radius dopants, dopant-driven and carrier-driven inhomogeneities, carrier-defect-lattice and carrier-lattice interactions and other factors are very important for the localization of carriers in hole-doped cuprates. In the present paper we study the extrinsic (defect-assisted) and intrinsic (phonon-assisted) single particle and pair selftrapping (ST) of carriers using the continuum model of ionic crystal and adiabatic approximation. The possibility of the formation of localized extrinsic and intrinsic (bi)polaronic states as well as hydrogenlike impurity states in charge-transfer (CT) gap of the cuprates is examined and compared with the experimental data.

\section{Calculation of the ground-state energy of the system of a defect- bound hole carrier in the polar crystal}

Electron-phonon interaction (EPI) affects the electronic properties of semiconductors and polar crystals in various ways depending on the strength of the electron-phonon interaction. Among them, the polaron formation and the ST are its typical and important effects on the carriers. The relevant charge carriers in hole-doped cuprates are large polarons $[13,18]$ and the strong EPIs are responsible for enhancement of the polaron mass $m_{\mathrm{p}}=(2.0-3.0) m_{\mathrm{h}}$ [19] (where $m_{\mathrm{h}}=m_{\mathrm{e}}$ is the free electron mass). According to Toyozawa [20], the mechanisms for ST of carriers are classified as intrinsic and extrinsic ones. The intrinsic mechanism means that carriers are self-trapped at deformed lattice sites through EPI. The extrinsic mechanism consists of short-range and/or long-range potentials by impurities or defects enhancing ST of carriers due to EPI. So far, there are no detailed quantitative studies of the intrinsic and extrinsic ST of the carriers in lightly doped cuprates. In order to better understand the situation, the possibility of the formation of extrinsic self-trapped states and of the intrinsic ones in the CT gap of the cuprates need to be thoroughly examined and compared with the existing experimental results confirming the existence of such localized in-gap states.

We use a continuum model proposed in [20] and adiabatic approximation to calculate the groundstate energy of an interacting system of a defect (dopant)-bound hole carrier in the polar crystal. In the continuous approximation, the functionals of the total energies of the single-carrier and two-carrier systems can be written as follows:

$$
\begin{aligned}
E_{1}[\psi(r)]= & \frac{\hbar^{2}}{2 m^{*}} \int[\nabla \psi(r)]^{2} \mathrm{~d}^{3} r-\frac{e^{2}}{2 \tilde{\varepsilon}} \int \frac{\psi^{2}(r) \psi^{2}\left(r^{\prime}\right)}{\left|r-r^{\prime}\right|} \mathrm{d}^{3} r \mathrm{~d}^{3} r^{\prime} \\
& -\frac{E_{\mathrm{d}}^{2}}{2 K} \int \psi^{4}(r) \mathrm{d}^{3} r-\frac{Z e^{2}}{\varepsilon_{0}} \int \frac{\psi^{2}(r)}{r} \mathrm{~d}^{3} r+\left(V_{0}-\frac{E_{\mathrm{d}} E_{\mathrm{dD}}}{K}\right) \int \psi^{2}(r) \delta(r) \mathrm{d}^{3} r
\end{aligned}
$$

and

$$
\begin{aligned}
E_{2}\left[\Psi\left(r_{1}, r_{2}\right)\right]= & \frac{\hbar^{2}}{2 m^{*}} \int\left[\left|\nabla_{1} \Psi\left(r_{1}, r_{2}\right)\right|^{2}+\left|\nabla_{2} \Psi\left(r_{1}, r_{2}\right)\right|^{2}\right] \mathrm{d}^{3} r_{1} \mathrm{~d}^{3} r_{2}+\frac{e^{2}}{\varepsilon_{\infty}} \int \frac{\Psi^{2}\left(r_{1}, r_{2}\right)}{\left|r_{1}-r_{2}\right|} \mathrm{d}^{3} r_{1} \mathrm{~d}^{3} r_{2} \\
& -\frac{2 e^{2}}{\tilde{\varepsilon}} \int \frac{\Psi^{2}\left(r_{1}, r_{2}\right) \Psi^{2}\left(r_{3}, r_{4}\right)}{\left|r_{1}-r_{3}\right|} \mathrm{d}^{3} r_{1} \mathrm{~d}^{3} r_{2} \mathrm{~d}^{3} r_{3} \mathrm{~d}^{3} r_{4} \\
& -\frac{2 E_{\mathrm{d}}^{2}}{K} \int \Psi^{2}\left(r_{1}, r_{2}\right) \Psi^{2}\left(r_{2}, r_{3}\right) \mathrm{d}^{3} r_{1} \mathrm{~d}^{3} r_{2} \mathrm{~d}^{3} r_{3}-\frac{2 Z e^{2}}{\varepsilon_{0}} \int \frac{\Psi^{2}\left(r_{1}, r_{2}\right)}{r_{1}} \mathrm{~d}^{3} r_{1} \mathrm{~d}^{3} r_{2} \\
& +2\left(V_{0}-\frac{E_{\mathrm{d}} E_{\mathrm{dD}}}{K}\right) \int \Psi^{2}\left(r_{1}, r_{2}\right) \delta\left(r_{1}\right) \mathrm{d}^{3} r_{1} \mathrm{~d}^{3} r_{2}
\end{aligned}
$$


where $m^{*}$ and $e$ are carrier effective mass and charge, respectively, $r_{1}$ and $r_{2}$ are the position vectors of the carriers, $E_{\mathrm{d}}$ and $E_{\mathrm{dD}}$ are the deformation potentials of the carrier and the defect, respectively, $\tilde{\varepsilon}$ is given by $\tilde{\varepsilon}^{-1}=\varepsilon_{\infty}^{-1}-\varepsilon_{0}^{-1}=(1-\eta) / \varepsilon_{\infty}, K$ is an elastic constant, $V_{0}$ is the short-range defect potential, $Z$ is the charge state of the defect. A large ionicity of the cuprates $\eta=\varepsilon_{\infty} / \varepsilon_{0} \ll 1$, enhances the polar EPI and the tendency to polaron formation.

In order to minimize the functionals (2.1) and (2.2) with respect to $\psi(r)$ and $\Psi\left(r_{1}, r_{2}\right)$ we can take the trial wave functions as follows:

$$
\psi(r)=N_{1} \exp [-(\sigma r)]
$$

and

$$
\Psi\left(r_{1}, r_{2}\right)=N_{2}\left[1+\gamma\left(\sigma r_{12}\right)\right] \exp \left[-\sigma\left(r_{1}+r_{2}\right)\right],
$$

where $N_{1}=\sigma^{3 / 2} / \sqrt{\pi}$ and $N_{2}=\sigma^{3} / \pi \sqrt{K_{1}(\gamma)}$ are the normalization factors, $\sigma=\beta / a_{0}, K_{1}(\gamma)=1+\frac{35}{8} \gamma+6 \gamma^{2}$ is the correlation coefficient, $\beta$ and $\gamma$ are the variational parameters that characterize the localization degree of carriers and the correlation in their motions, respectively. $r_{12}=\left|r_{1}-r_{2}\right|$ is the distance between the carriers, $a_{0}$ is the lattice constant. Substituting equations (2.3) and (2.4) into equations (2.1) and (2.2), and performing the integrations in equations (2.1) and (2.2), we obtain the following functionals

$$
E_{1}[\beta]=A\left[\beta^{2}-g_{\mathrm{s}}\left(1+b_{\mathrm{s}}\right) \beta^{3}-g_{1}\left(1-\eta+\frac{16}{5} Z \eta\right) \beta\right]
$$

and

$$
\begin{aligned}
E_{2}[\beta, \gamma]= & 2 A \frac{K_{2}(\gamma)}{K_{1}(\gamma)}\left\{\beta^{2}-\frac{8}{5} g_{1}\left[2(1-\eta) \frac{K_{4}(\gamma)}{K_{1}(\gamma) K_{2}(\gamma)}+Z \eta \frac{K_{5}(\gamma)}{K_{2}(\gamma)}-\frac{K_{3}(\gamma)}{K_{2}(\gamma)}\right] \beta\right. \\
& \left.-16 g_{\mathrm{s}}\left[\frac{K_{6}(\gamma)}{K_{1}(\gamma) K_{2}(\gamma)}+\frac{b_{\mathrm{s}}}{16} \frac{K_{7}(\gamma)}{K_{2}(\gamma)}\right] \beta^{3}\right\},
\end{aligned}
$$

where $A=\hbar^{2} / 2 m^{*} a_{0}^{2}, g_{\mathrm{s}}=E_{\mathrm{d}}^{2} / 16 \pi K a_{0}^{3} A$ and $g_{1}=5 e^{2} / 16 \varepsilon_{\infty} a_{0} A$ are dimensionless short-range and longrange carrier-phonon coupling parameters, $b_{s}=16\left(E_{\mathrm{dD}} / E_{\mathrm{d}}-K V_{0} / E_{\mathrm{d}}^{2}\right)$ is short-range carrier-defect-phonon coupling parameter, and the correlation coefficients are analytical functions of the variational parameter $\gamma$ :

$$
\begin{aligned}
& K_{2}(\gamma)=1+\frac{25}{8} \gamma+4 \gamma^{2}, \\
& K_{4}(\gamma)=\frac{5}{8}+\frac{1087}{216} \gamma+\frac{38237}{2304} \gamma^{2}+\frac{67639}{2592} \gamma^{3}+\frac{4293}{256} \gamma^{4}, \\
& K_{3}(\gamma)=\frac{5}{8} \gamma+2 \gamma+\frac{35}{16} \gamma^{2}, \quad K_{5}(\gamma)=2+\frac{15}{2} \gamma+9 \gamma^{2}, \\
& K_{6}(\gamma)=\frac{1}{8}+\frac{185}{216} \gamma+\frac{4199}{1728} \gamma^{2}+\frac{8591}{2592} \gamma^{3}+\frac{477}{256} \gamma^{4}, \\
& K_{7}(\gamma)=1+3 \gamma+3 \gamma^{2} .
\end{aligned}
$$

Minimization of the functionals (2.5) and (2.6) over the variational parameters $\beta$ and $\gamma$ would give the ground state energies $E_{1}^{\min }(\beta)$ and $E_{2}^{\min }(\beta, \gamma)$ of single and pair self-trapped carriers. As a function of $\beta$ and $\gamma$, these functionals have also got the maxima $E_{1}^{\max }(\beta)$ and $E_{2}^{\max }(\beta, \gamma)$. The other parameters $A$, $g_{\mathrm{s}}, g_{\mathrm{l}}, b_{\mathrm{s}}$ and $Z$ entering equations (2.5) and (2.6) play different roles in the formation of self-trapped states and determine the nature of the localized states of carriers in doped polar materials. The values of the parameters $A, g_{\mathrm{s}}$ and $g_{1}$ can be obtained using the experimental values of the parameters $a_{0}, m^{*}$, $K, \varepsilon_{\infty}, \varepsilon_{0}$ and the Fermi energy $E_{\mathrm{F}}$ of the undoped cuprates. The parameters $b_{\mathrm{s}}$ and $Z$ characterize the formation of extrinsic (i.e., hydrogenic and non-hydrogenic) self-trapped states of carriers, whereas the parameters $g_{s}$ and $g_{1}$ characterizing the strengths of short- and long-range carrier-phonon interactions are responsible for the formation of intrinsic self-trapped states.

Using the equations (2.5) and (2.6), we calculate the energies of different localized in-gap states in the CT gap of the cuprates. In order to determine the nature of these in-gap states and the quasi-free to localized state transition, we distinguish different physical situations in these systems. One can make 
interesting analyses of real systems based on the sign and magnitude of $b_{\mathrm{s}}$. In hole-doped cuprates, the situations might be quite different for different types of dopants. In particular, the signs of the deformation potential constants $E_{\mathrm{d}}$ and $E_{\mathrm{dD}}$ for holes and small-radius defects are always positive, while $E_{\mathrm{dD}}$ for large-radius defects is negative [20]. However, at present no information is available for the magnitudes of the parameters $E_{\mathrm{dD}}$ and $V_{0}$. Therefore, the parameter $b_{\mathrm{s}}$ in equations (2.5) and (2.6) can be considered as the free parameter. We consider first the possibility of the formation of localized in-gap states at single and pair ST of carriers near the small-radius dopants (with $E_{\mathrm{dD}}>0$ or $b_{\mathrm{s}}>0$ ) in La-based cuprates. In this case, both short and long range parts of the defect potential in equations (2.1) and (2.2) are attractive, so that the substitution of small-radius cations (e.g., $\mathrm{Ca}^{2+}$ and $\mathrm{Nd}^{3+}$ ions) for $\mathrm{La}^{3+}$ ions in $\mathrm{La}_{2} \mathrm{CuO}_{4}$ and for $\mathrm{Sr}^{2+}$ ions in LSCO leads to a combined defect- and phonon-assisted ST of hole carriers with the formation of localized single-carrier and two-carrier impurity states, which are extrinsic polaronic and bipolaronic (the so-called U-pairing) states. At $Z \neq 0$ and $b_{\mathrm{s}}>0$, the minima of $E_{1}(\beta)$ and $E_{2}(\beta, \gamma)$ correspond to the ground-state energies of the extrinsic large polaron and bipolaron, respectively, measured with respect to the top of the oxygen valence band. The binding energies of such extrinsic large polaron and bipolaron (or negative U center) are defined as $E_{\mathrm{pI}}=\left|E_{1}^{\min }(\beta)\right|$ and $E_{\mathrm{bU}}=\left|E_{2}^{\min }(\beta, \gamma)-2 E_{1}^{\min }(\beta)\right|$, respectively. In $3 \mathrm{D}$ systems, there is generally a potential barrier between the large- and small-radius self-trapped states. The two states of the extrinsic large polaron are separated by a potential barrier, with activation energy $E_{1}^{\mathrm{A}}=E_{1}^{\max }(\beta)-E_{1}^{\min }(\beta)$ needed for the transition from the large-radius localized state to the small-radius one. The potential barrier $E_{2}^{\mathrm{A}}=E_{2}^{\max }(\beta, \gamma)-E_{2}^{\min }(\beta)$ exists between the large and small-radius extrinsic bipolaronic states.

We now calculate the basic parameters of the extrinsic large (bi)polarons in La-based cuprates. At low temperature, the La-based cuprates are orthorhombic with the lattice parameter $a_{0} \simeq 5.4 \AA$. According to the spectroscopy data, the Fermi energy of the undoped cuprates is about $E_{\mathrm{F}} \simeq 7 \mathrm{eV}$ [21]. To determine the value of the short-range carrier-phonon coupling constant $g_{s}$, we can estimate the deformation potential $E_{\mathrm{d}}$ as $E_{\mathrm{d}}=(2 / 3) E_{\mathrm{F}}$ [22]. For the cuprates, typical values of other parameters are $m^{*}=m_{\mathrm{e}}$ [13], $\varepsilon_{\infty}=3-5$ [23, 24], $K=1.4 \cdot 10^{12} \mathrm{dyn} / \mathrm{cm}^{2}$ [25], and $Z=1$. The calculated values of $E_{\mathrm{pI}}, E_{\mathrm{bU}}, E_{1}^{\mathrm{A}}$ and $E_{2}^{\mathrm{A}}$ for $b_{\mathrm{s}}=1$ and different values of $\varepsilon_{\infty}$ and $\eta$ are presented in table 1. From table 1 we can see that the potential barriers separating the large- and small-radius extrinsic (bi)polaronic states are rather high. These high potential barriers prevent the formation of small extrinsic (bi)polarons in 3D cuprates. The defect- and phonon-assisted ST of large polaron and large bipolaron in La-based cuprates are shown in figures 1 and 2 respectively.

Table 1. The calculated parameters of the extrinsic large polarons and bipolarons (with correlation between the pairing carriers) in 3D cuprates at $Z=1, b_{\mathrm{s}}=1$ and different values of $\varepsilon_{\infty}$ and $\eta$.

\begin{tabular}{|c|c|c|c|c|c|c|c|c|c|c|c|c|}
\hline \multirow{2}{*}{$\eta$} & \multicolumn{4}{|c|}{$\overline{\varepsilon_{\infty}=3.5}$} & \multicolumn{4}{|c|}{$\overline{\overline{\varepsilon_{\infty}=4}}$} & \multicolumn{4}{|c|}{$\varepsilon_{\infty}=4.5$} \\
\hline & $E_{\mathrm{pI}}, \mathrm{eV}$ & $E_{\mathrm{bU}}, \mathrm{eV}$ & $E_{1}^{\mathrm{A}}, \mathrm{eV}$ & $E_{2}^{\mathrm{A}}, \mathrm{eV}$ & $E_{\mathrm{pI}}, \mathrm{eV}$ & $E_{\mathrm{bU}}, \mathrm{eV}$ & $E_{1}^{\mathrm{A}}, \mathrm{eV}$ & $E_{2}^{\mathrm{A}}, \mathrm{eV}$ & $E_{\mathrm{pI}}, \mathrm{eV}$ & $E_{\mathrm{bU}}, \mathrm{eV}$ & $E_{1}^{\mathrm{A}}, \mathrm{eV}$ & $E_{2}^{\mathrm{A}}, \mathrm{eV}$ \\
\hline 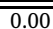 & 0.1135 & 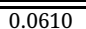 & 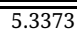 & $\overline{5.8611}$ & $\overline{c 0.0863}$ & 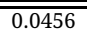 & 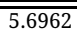 & 26.4752 & 0.0679 & 0.0354 & 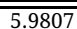 & 86.7958 \\
\hline 0.02 & 0.1240 & 0.0525 & 5.2128 & 5.7649 & 0.0943 & 0.0391 & 5.5849 & 6.3881 & 0.0741 & 0.0303 & 5.8801 & 6.7090 \\
\hline 0.04 & 0.1349 & 0.0434 & 5.0893 & 5.6693 & 0.1026 & 0.0321 & 5.4742 & 6.3013 & 0.0806 & 0.0247 & 5.7800 & 6.6224 \\
\hline 0.06 & 0.1464 & 0.0336 & 4.9668 & 5.5743 & 0.1113 & 0.0246 & 5.3643 & 6.2150 & 0.0874 & 0.0188 & 5.6804 & 6.5359 \\
\hline 0.08 & 0.1584 & 0.0231 & 4.8453 & 5.4799 & 0.1203 & 0.0166 & 5.2552 & 6.1292 & 0.0945 & 0.0125 & 5.5815 & 6.4495 \\
\hline 0.10 & 0.1709 & 0.0120 & 4.7248 & 5.3861 & 0.1298 & 0.0081 & 5.1468 & 6.0438 & 0.1019 & 0.0058 & 5.4831 & 6.3633 \\
\hline 0.12 & 0.1839 & 0.0001 & 4.6053 & 5.2929 & 0.1396 & - & 5.0391 & 5.9588 & 0.1096 & - & 5.3854 & 6.2773 \\
\hline
\end{tabular}

Another interesting question is in what way large-radius dopants in cuprates affect the carrier-phonon system, especially near such defects. This opposite situation is realized in LSCO or $\mathrm{La}_{2-x} \mathrm{Ba}_{x} \mathrm{CuO}_{4}$ (LBCO), where the radius of $\mathrm{Sr}^{2+}$ ions is larger than that of $\mathrm{La}^{3+}$ ions [26], so that for $\mathrm{Sr}^{2+}$ ion $Z=1$, $E_{\mathrm{dD}}<0$ or $b_{s}<0$. In this case, the short-range part of the impurity potential in equation (2.1) is repulsive. Therefore, one can treat it like a hard core. The hole-lattice interactions near the large-radius dopants in LSCO and LBCO are suppressed by this repulsive defect potential and hole carriers are localized at a distance from the dopants (i.e., hole-carriers are loosely bound to dopants by long-range Coulomb attraction). From these considerations, it follows that the hole-lattice interaction near the largeradius dopants is weak and the localized impurity state may be of a hydrogen-like character described by a rigid lattice model [27]. Therefore, we can consider the hydrogen-like impurity centers having the 


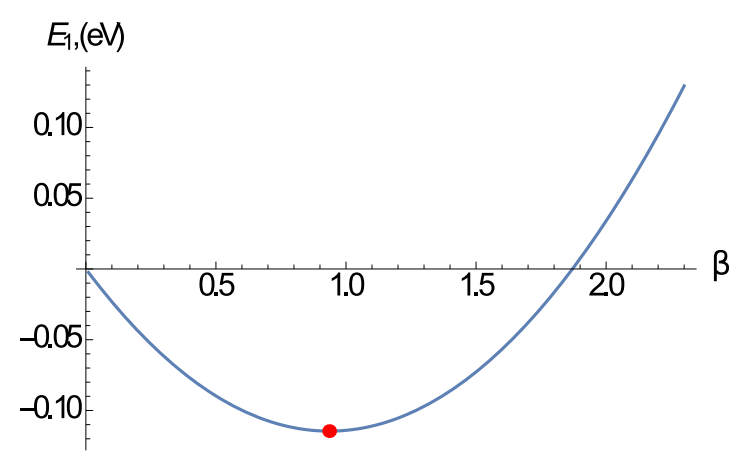

Figure 1. (Color online) The dependence of ground-state energy of extrinsic large polaron on the variational parameter $\beta$ for $\eta=0.08$ in La-based cuprates. The red point indicates a single carrier self-trapped state.

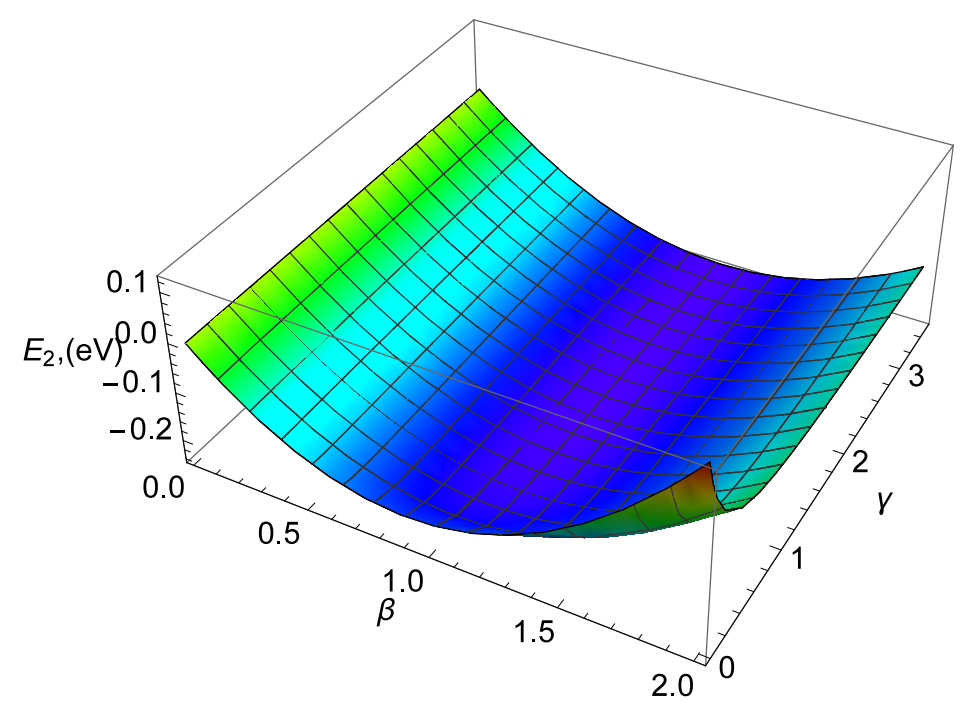

Figure 2. (Color online) The dependence of ground-state energy of extrinsic large bipolaron on the variational parameters $\beta$ and $\gamma$ for $\eta=0.08$ in La-based cuprates.

Bohr radius $a_{\mathrm{H}}=0.529 \varepsilon_{0}\left(m_{\mathrm{e}} / m^{*}\right) \AA$ and the ionization energy $E_{\mathrm{I}}^{\mathrm{H}}=e^{2} / 2 \varepsilon_{0} a_{\mathrm{H}}$ in lightly doped LSCO and LBCO. A similar argument can be made for other cuprates, such as $\mathrm{La}_{2} \mathrm{CuO}_{4+\delta}, \mathrm{YBa}_{2} \mathrm{Cu}_{3} \mathrm{O}_{7-\delta}(\mathrm{YBCO})$ and $\mathrm{Bi}_{2} \mathrm{Sr}_{2} \mathrm{CaCu}_{2} \mathrm{O}_{8+\delta}$ (Bi-2212). In these systems, the doping centers are excess oxygen atoms which capture the electrons from the oxygen conduction band and form the acceptor centers, i.e., $\mathrm{O}^{-}$ions. One can assume that such extra $\mathrm{O}^{-}$ions just like $\mathrm{Sr}^{2+}$ ions may have negative $E_{\mathrm{dD}}$.

Figure 3 shows the variation of the ratio $E_{\mathrm{bU}} / 2 E_{\mathrm{pI}}$ with $\eta$ for $b_{\mathrm{s}}=0.5$ and 2.0 for the stability region of the extrinsic large bipolaron in 3D cuprates. One can find out that such bipolarons exist as long as $\eta$ is less than the critical value $\eta_{\mathrm{c}}=0.127$ and the ratio $E_{\mathrm{bU}} / 2 E_{\mathrm{pI}}$ reaches up to 0.287 (at $\varepsilon_{\infty}=3$ and $\eta \rightarrow 0$ ). We have determined the stability region of the extrinsic large bipolaron in cuprates and found that such bipolarons exist as long as $\eta$ is less than the critical value $\eta_{\mathrm{c}}=0.127$ and the ratio $E_{\mathrm{bU}} / 2 E_{\mathrm{pI}}$ reaches up to 0.287 (at $\varepsilon_{\infty}=3$ and $\eta \rightarrow 0$ ). Figure 4 shows the variation of the ratio $E_{\mathrm{bB}} / 2 E_{\mathrm{p}}$ with $\eta$ for $\varepsilon_{\infty}=3,4$ and 5 for real large bipolarons in cuprates. One can see that in 3D cuprates, the intrinsic large bipolarons can exist at $\eta=\eta_{\mathrm{c}} \leqslant 0.138$ and the ratio $E_{\mathrm{bB}} / 2 E_{\mathrm{p}}$ reaches up to 0.27 (at $\varepsilon_{\infty}=3$ and $\eta \rightarrow 0$ ). While the inset in figure 4 shows the variation of the ratio $E_{\mathrm{bB}} / 2 E_{\mathrm{p}}$ [calculated from equations (2.5) and (2.6) at $g_{\mathrm{s}}=0$ and $Z=0$ ] with $\eta$ for large optical bipolaron. Of course, besides the ratio $\eta=\varepsilon_{\infty} / \varepsilon_{0}$, the values of the Fröhlich electron-phonon coupling constant $\alpha$ are very important for the formation of large optical bipolarons. The long-range coupling of carriers with optical phonons is much stronger than their shortrange coupling with acoustic phonons. Therefore, the long-range Fröhlich-type EPI in polar materials 


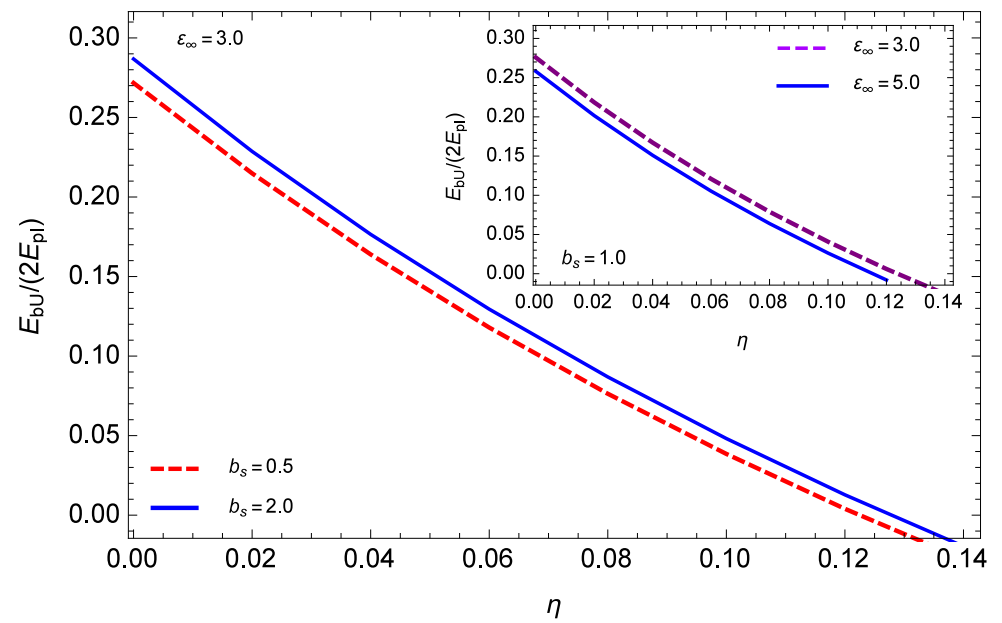

Figure 3. (Color online) The ratio of the binding energy of the extrinsic large bipolaron to twice the extrinsic large polaron binding energy as a function of $\eta$ for two values of $b_{\mathrm{S}}$ in 3D cuprates. The inset shows the dependence of the ratio of the binding energy of the extrinsic large bipolaron to twice that of the extrinsic large polaron on $\eta$ for two values of $\varepsilon_{\infty}$ in 3D cuprates.

have been studied extensively [28, 29], although the short-range deformation potential type interaction is also important and leads to new effects. The dimentionless EPI Fröhlich coupling constant is defined as

$$
\alpha=\frac{e^{2}(1-\eta)}{2 \varepsilon_{\infty} \hbar \omega_{\mathrm{LO}}}\left(\frac{2 m^{*} \omega_{\mathrm{LO}}}{\hbar}\right)^{1 / 2}
$$

where $\omega_{\mathrm{LO}}$ is the frequency of the longitudinal-optical (LO) phonon in an ionic crystal.

In polar materials, the formation of optical bipolarons is favored by larger values of $\alpha$ and by smaller values of $\eta[2,29]$, i.e., the optical (Fröhlich or Pekar) bipolarons exist only if $\alpha$ is is greater than a critical value $\alpha_{\mathrm{c}}$ and when $\eta<\eta_{\mathrm{c}}$. Such 3D bipolarons can exist above rather high critical values $\alpha_{\mathrm{c}}$, e.g., $\alpha_{\mathrm{c}}=7.3$ as found by Adamowski [30] and $\alpha_{\mathrm{c}}=6.8$ found by Verbist, Peeters and Devreese [31]. Further, the value

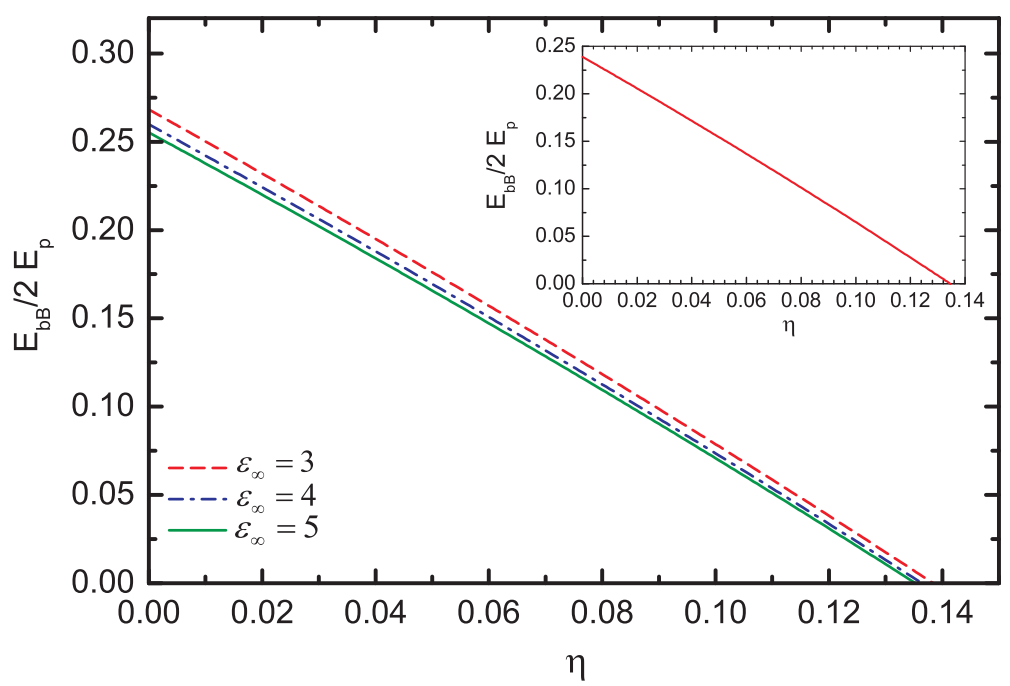

Figure 4. (Color online) The ratio of the binding energy of the real large bipolaron to twice that of the real large polaron as a function of $\eta$ for different values of $\varepsilon_{\infty}$ in 3D cuprates. The inset illustrates the ratio of the binding energy of the optical large bipolaron to twice that of the optical large polaron on $\eta$ for $\varepsilon_{\infty}=3.0$ in 3D cuprates. 
of $\alpha_{\mathrm{c}}$ corresponding to the onset of the strong coupling regime is found to be $\alpha_{\mathrm{c}}=5.8$ [32], below which the formation of large optical bipolaron in 3D systems is unlikely. At a given value of $\eta$, the value of $\alpha_{\mathrm{c}}$ depends on $m^{*}, \varepsilon_{\infty}$ and $\omega_{\mathrm{LO}}$. The values of $\hbar \omega_{\mathrm{LO}}$ in high- $T_{\mathrm{c}}$ cuprates range from 0.03 to $0.05 \mathrm{eV}$ [13, 33]. Then, according to equation (2.7), the values of $\alpha$ corresponding to these high- $T_{\mathrm{c}}$ materials with $m^{*}=m_{\mathrm{e}}, \varepsilon_{\infty}=3$ and $\eta=0.02-0.10$ are equal to $\alpha=4.96-6.95$. Thus, the conditions for the formation of large optical bipolarons are more favorable in the cuprates with $\varepsilon_{\infty}=3, \eta=0.02-0.06$ and $\hbar \omega_{\mathrm{LO}}=$ 0.03-0.04 eV, at which conditions $\alpha>\alpha_{\mathrm{c}} \simeq 5.8$ [32] and $\alpha>\alpha_{\mathrm{c}}=6.8$ [31] are well satisfied.

We note here that the largest values of $\eta_{\mathrm{c}}=0.079-0.14$ and $E_{\mathrm{bB}} / 2 E_{\mathrm{p}}=0.22-0.25$ as found in the literature [34-36] (see also [29]) were obtained for the optical bipolaron and do not correspond to specific substances. At the same time, the critical values of $\eta$ below which the optical bipolarons can exist in cuprates were small enough $\left(\eta_{\mathrm{c}}=0.040-0.055\right)$, as estimated in [29]. Our results are quite impressive in the sense that both the real and the optical bipolaron in 3D cuprates, can really exist for relatively large values of $\eta$ (figure 4) and the large bipolarons are formed with the binding energies reaching up to $27 \%$ (at $\eta \rightarrow 0$ ) of twice the large polaron energy. The distinctive feature of the cuprates is their very large ratio of static to high-frequency dielectric constants. This situation is favorable for carriers attracted to polarization well created by the other ones or to Coulomb centers (dopants) to form 3D intrinsic or extrinsic large bipolarons. At $\varepsilon_{0}>30$, such bipolarons (pair states) can be formed in lightly doped cuprates and they become unstable in an underdoped regime. As can be seen from tables 1 and 2 the binding energies of extrinsic and intrinsic large (bi)polarons and the ratios $E_{\mathrm{bU}} / 2 E_{\mathrm{pI}}$ and $E_{\mathrm{bB}} / 2 E_{\mathrm{p}}$ depend on several parameters. In particular, $E_{\mathrm{pI}}, E_{\mathrm{p}}, E_{\mathrm{bU}}$ and $E_{\mathrm{bB}}$ would rapidly increase with $\varepsilon_{\infty}$ decreasing from 5 to 3 , while the ratios $E_{\mathrm{bU}} / 2 E_{\mathrm{pI}}$ and $E_{\mathrm{bB}} / 2 E_{\mathrm{p}}$ increase more slowly as $\varepsilon_{\infty}$ decreases. Interestingly, $E_{\mathrm{pI}}$ is an increasing function of $\eta$ (table 1), while $E_{\mathrm{p}}$ is a decreasing function of $\eta$ (table2). Furthermore, we find that both $E_{\mathrm{bU}}$ and $E_{\mathrm{bB}}$ decrease with increasing $\eta$. We also find that the ratio $E_{\mathrm{bU}} / 2 E_{\mathrm{pI}}$ appreciably increases with $b_{\mathrm{S}}$ as shown in figure 3 . The calculated values of the radii of extrinsic large (bi)polarons $R_{\mathrm{I}}\left(R_{\mathrm{BI}}\right)$ (at $Z=1$ and $b_{\mathrm{S}}=1$ ) and intrinsic large (bi)polarons, $R_{\mathrm{p}}\left(R_{\mathrm{B}}\right)$ (at $Z=0$ and $b_{\mathrm{s}}=0$ ) for different values of $\varepsilon_{\infty}$ and $\eta$ are presented in table 3 .

Table 2. Calculated parameters of the real large polarons and bipolarons (with correlation between the pairing carriers) in 3D cuprates at different values of $\varepsilon_{\infty}$ and $\eta$.

\begin{tabular}{|c|c|c|c|c|c|c|c|c|c|c|c|c|}
\hline \multirow{2}{*}{$\eta$} & \multicolumn{4}{|c|}{$\varepsilon_{\infty}=3.5$} & \multicolumn{4}{|c|}{$\varepsilon_{\infty}=4$} & \multicolumn{4}{|c|}{$\varepsilon_{\infty}=4.5$} \\
\hline & $E_{\mathrm{p}}, \mathrm{eV}$ & $E_{\mathrm{bB}}, \mathrm{eV}$ & $E_{\mathrm{p}}^{\mathrm{A}}, \mathrm{eV}$ & $E_{\mathrm{B}}^{\mathrm{A}}, \mathrm{eV}$ & $E_{\mathrm{p}}, \mathrm{eV}$ & $E_{\mathrm{bB}}, \mathrm{eV}$ & $E_{\mathrm{p}}^{\mathrm{A}}, \mathrm{eV}$ & $E_{\mathrm{B}}^{\mathrm{A}}, \mathrm{eV}$ & $E_{\mathrm{p}}, \mathrm{eV}$ & $E_{\mathrm{bB}}, \mathrm{eV}$ & $E_{\mathrm{p}}^{\mathrm{A}}, \mathrm{eV}$ & $E_{\mathrm{B}}^{\mathrm{A}}, \mathrm{eV}$ \\
\hline 0.00 & 0.1107 & 0.0583 & 27.275 & 19.989 & 0.0845 & 0.0439 & 28.049 & 21.063 & 0.0666 & 0.0343 & 28.656 & 21.744 \\
\hline 0.02 & 0.1063 & 0.0484 & 27.398 & 20.269 & 0.0811 & 0.0364 & 28.158 & 21.313 & 0.0640 & 0.0284 & 28.753 & 21.980 \\
\hline 0.04 & 0.1019 & 0.0389 & 27.521 & 20.551 & $\begin{array}{c}0.0778 \\
\end{array}$ & 0.0293 & 28.267 & 21.564 & 0.0614 & 0.0228 & 28.851 & 22.217 \\
\hline 0.06 & 0.0977 & 0.0299 & 27.645 & 20.834 & 0.0746 & 0.0225 & 28.376 & 21.815 & 0.0588 & 0.0175 & 28.949 & 22.453 \\
\hline 0.08 & 0.0935 & 0.0215 & 27.769 & 21.118 & 0.0714 & 0.0161 & 28.485 & 22.068 & 0.0563 & 0.0125 & 29.046 & 22.690 \\
\hline 0.10 & 0.0895 & 0.0136 & 27.893 & 21.404 & 0.0683 & 0.0101 & 28.595 & 22.321 & 0.0539 & 0.0078 & 29.144 & 22.928 \\
\hline 0.12 & 0.0855 & 0.0061 & 28.017 & 21.691 & 0.0653 & 0.0044 & 28.704 & 22.576 & 0.0515 & 0.0003 & 29.242 & 23.166 \\
\hline 0.14 & 0.0816 & - & 28.142 & 21.980 & 0.0623 & - & 28.814 & 22.832 & 0.0491 & - & 29.341 & 23.404 \\
\hline
\end{tabular}

Table 3. Calculated values of the radii of intrinsic and extrinsic large (bi)polarons $R_{\mathrm{p}}, R_{\mathrm{I}}, R_{\mathrm{B}}$ and $R_{\mathrm{BI}}$ in 3D cuprates at different values of $\varepsilon_{\infty}$ and $\eta$.

\begin{tabular}{|c|c|c|c|c|c|c|c|c|c|c|c|c|}
\hline \multirow{2}{*}{$\eta$} & \multicolumn{4}{|c|}{$\varepsilon_{\infty}=3.5$} & \multicolumn{4}{|c|}{$\varepsilon_{\infty}=4$} & \multicolumn{4}{|c|}{$\varepsilon_{\infty}=4.5$} \\
\hline & $R_{\mathrm{p}}, \AA$ & $R_{\mathrm{B}}, \AA$ & $R_{\mathrm{I}}, \AA$ & $R_{\mathrm{BI}}, \AA$ & $R_{\mathrm{p}}, \AA$ & $R_{\mathrm{B}}, \AA$ & $R_{\mathrm{I}}, \AA$ & $R_{\mathrm{BI}}, \AA$ & $R_{\mathrm{p}}, \AA$ & $R_{\mathrm{B}}, \AA$ & $R_{\mathrm{I}}, \AA$ & $R_{\mathrm{BI}}, \AA$ \\
\hline 0.00 & 8.6096 & 13.045 & 8.2839 & 12.403 & 9.8840 & 15.057 & 9.5633 & 14.434 & 11.158 & 17.066 & 10.841 & 16.457 \\
\hline 0.02 & 8.7917 & $\begin{array}{l}13.577 \\
\end{array}$ & 7.9061 & 12.084 & 10.092 & 15.663 & 9.1322 & 14.072 & 11.392 & 17.746 & 10.357 & 16.052 \\
\hline 0.04 & $\begin{array}{l}8.9813 \\
\end{array}$ & 14.146 & 7.5586 & 11.777 & 10.309 & 16.311 & 8.7357 & 13.724 & 11.636 & 18.475 & 9.9111 & 15.662 \\
\hline 0.06 & $\begin{array}{l}9.1791 \\
\end{array}$ & 14.756 & 7.2378 & 11.482 & 10.535 & 17.007 & 8.3699 & 13.390 & 11.890 & 19.256 & 9.5001 & 15.288 \\
\hline 0.08 & 9.3854 & 15.413 & 6.9409 & 11.199 & 10.770 & 17.756 & 8.0313 & 13.068 & 12.155 & 20.098 & 9.1197 & 14.928 \\
\hline 0.10 & 9.6009 & 16.121 & 6.6652 & 10.926 & 11.017 & 18.564 & 7.7169 & 12.758 & 12.432 & 21.005 & 8.7665 & 14.581 \\
\hline 0.12 & 9.8261 & 16.887 & 6.4084 & 10.662 & 11.274 & 19.438 & 7.4243 & 12.460 & 12.722 & 21.986 & 8.4379 & 14.248 \\
\hline 0.14 & 10.062 & $\begin{array}{l}17.718 \\
\end{array}$ & 6.1688 & 10.408 & 11.543 & 20.386 & 7.1512 & 12.173 & 13.025 & 23.051 & 8.1312 & 13.926 \\
\hline
\end{tabular}




\section{Discussion}

We now make some remarks about the characteristic (i.e., binding) energies of large bipolarons in the cuprates. The binding energies of extrinsic and intrinsic bipolarons strongly depend on $\varepsilon_{\infty}, \varepsilon_{0}$ and $\eta$. The values of $\varepsilon_{\infty}$ and $\varepsilon_{0}$ observed in high- $T_{\mathrm{c}}$ cuprates are 3-5 [4, 37, 38] and 33-105 [4, 39], respectively, so that the values of $\eta$ range from 0.028 to 0.15 . Using the values of $\varepsilon_{\infty}=3$ and $\eta \simeq 0.03$, we find $E_{\mathrm{bU}} \simeq 0.07 \mathrm{eV}$ and $E_{\mathrm{bB}} \simeq 0.061 \mathrm{eV}$. If we take other experimental values of $\varepsilon_{\infty}=4$ and $\varepsilon_{0} \simeq 50$ for the cuprates (see [2, 29]), then we obtain $E_{\mathrm{bU}} \simeq 0.017 \mathrm{eV}$ and $E_{\mathrm{bB}} \simeq 0.016 \mathrm{eV}$ at $\eta=0.08$. Further, at $\varepsilon_{\infty}=5$ and $\eta=0.08$ we find $E_{\mathrm{bU}} \simeq 0.0097 \mathrm{eV}$ and $E_{\mathrm{bB}} \simeq 0.0102 \mathrm{eV}$. Thus, the extrinsic and intrinsic bipolarons can be experimentally found in high- $T_{\mathrm{c}}$ cuprates in the energy ranges $\sim 0.01-0.07 \mathrm{eV}$ and $\sim 0.01-0.06 \mathrm{eV}$, respectively. The binding energies of large polarons and bipolarons are manifested in the excitation spectra of the hole-doped cuprates as the temperature-independent low-energy gaps or pseudogaps, which are different from the high-energy CT gaps $\left(\Delta_{\mathrm{CT}} \simeq 1.5-2.0 \mathrm{eV}[13]\right)$ of the cuprates.

It is of interest to compare our results with experimental data on localized in-gap states (or bands) and energy gaps (which are responsible for the existance of insulating phase and are precursors to the pseudogaps observed in the metallic state) in hole-doped cuprates. The above extrinsic and intrinsic (bi)polaronic states as well as hydrogenic impurity states emerge in the CT gap of the cuprates. In the experiments, these localized states are displayed as the in-gap states. One can see that the value of $E_{\mathrm{pI}} \simeq 0.13 \mathrm{eV}$ obtained at $\varepsilon_{\infty}=4$ and $\eta=0.1$ (table 1) is consistent with experimental data for lightly doped $\mathrm{La}_{2} \mathrm{CuO}_{4+\delta}$ [13]. The in-gap impurity band observed in this system at $0.13 \mathrm{eV}$ might be associated with the extrinsic large polarons. While the values of $E_{\mathrm{p}} \simeq 0.096-0.105 \mathrm{eV}$ (table 2) obtained at $\varepsilon_{\infty}=3.5$ and $\eta=0.04-0.06$ agree reasonably well with the large pseudogap value $\sim 0.1 \mathrm{eV}$ observed in LSCO [40]. One of the important experimental observations is that in LSCO, the flatband [41], which is $\sim 0.12 \mathrm{eV}$ below the Fermi energy for $x=0.05$, moves upwards monotonously with increasing $x$, but the flatband is lowered as $x$ decreases and loses its intensity in the insulating phase. Apparently, the flatband observed by ARPES in the lightly doped LSCO $(x=0.05)$ is the energy band of large polarons since the effective mass of carriers obtained from the analysis of the ARPES spectra is about $2.1 m_{\mathrm{e}}$ [41]. The values of $R_{\mathrm{p}}$ (table 3) are also in good agreement with the experimental values of the radii of polarons which vary from 6 to $10 \AA$ in cuprates [13].

\section{Conclusions}

We have studied the possible mechanisms of carrier localization in inhomogeneous hole-doped cuprates. The quantitative theory of the impurity-assisted and phonon-assisted single particle and pair ST of hole carriers in 3D lightly doped cuprates is developed within the continuum model and adiabatic approximation. The possible mechanisms for carrier localization lead to the formation of extrinsic large (bi)polaronic states, the hydrogenic impurity states (i.e., impurities with loosely bound free carriers or large polarons) and intrinsic large (bi)polaronic states in the CT gap of the cuprates. We have variationally calculated the binding energies and radii of the extrinsic and intrinsic large polarons and bipolarons, taking into account the short- and long-range parts of the carrier-defect-phonon and carrier-phonon interactions. We have determined the stability region of the extrinsic large bipolaron in cuprates and found that such bipolarons exist as long as $\eta$ is less than the critical value $\eta_{\mathrm{c}}=0.127$ and the ratio $E_{\mathrm{bU}} / 2 E_{\mathrm{pI}}$ (where $E_{\mathrm{pI}}$ and $E_{\mathrm{bU}}$ are the binding energies of the extrinsic large polaron and bipolaron, respectively) reaches 0.287 (at $\varepsilon_{\infty}=3$ and $\eta \rightarrow 0$ ). We have obtained the conditions for the real large bipolaron stability and estimated the values of $E_{\mathrm{bB}} / 2 E_{\mathrm{p}}$ in $3 \mathrm{D}$ cuprates (where $E_{\mathrm{p}}$ and $E_{\mathrm{bB}}$ are the binding energies of the intrinsic large polaron and bipolaron, respectively).

\section{Acknowledgements}

This work was supported by the Foundation of National University of Uzbekistan under Grant No: F2-FA-F115. 


\section{References}

1. Polarons and Bipolarons in High- $\mathrm{T}_{\mathrm{c}}$ Superconductors and Related Materials, Salje E.K.H., Alexandrov A.S., Liang W.Y. (Eds.), Cambridge University Press, Cambridge, 2005; doi 10.1002/adma.19970090521.

2. Devreese J.T., Alexandrov A.S., Rep. Prog. Phys., 2009, 72, 066501; doi 10.1088/0034-4885/72/6/066501.

3. Mishchenko A.S., Nagaosa N., Shen K.M., Shen Z.-X., Zhou X.J., Devereaux T.P., EPL, 2011, 95, 57007; doi 10.1209/0295-5075/95/57007

4. Imada M., Fujimori A., Tokura Y., Rev. Mod. Phys., 1998, 70, 1039; doi 10.1103/RevModPhys.70.1039.

5. Damascelli A., Hussian Z., Shen Z.-X., Rev. Mod. Phys., 2003, 75, 473; doi 10.1103/RevModPhys.75.473

6. Andreson P.W., The Theory of Superconductivity in the High- $\mathrm{T}_{\mathrm{C}}$ Cuprates, Princeton University Press, Princeton, 1997.

7. Anderson P.W., Science, 2007, 316, 1705; doi 10.1126/science.1140970

8. Alexandrov A.S., J. Phys.: Condens. Matter, 2007, 19, 125216; doi 10.1088/0953-8984/19/12/125216

9. Alexandrov A.S., Phys. Rev. B, 1996, 53, 2863; doi 10.1103/PhysRevB.53.2863.

10. Mishchenko A.S., Nagaosa N., Phys. Rev. Lett., 2004, 93, 036402; doi 10.1103/PhysRevLett.93.036402.

11. Mishchenko A.S., Nagaosa N., Shen Z.-X., De Filippis G., Cataudella V., Devereaux T.P., Bernhard C., Kim K.W., Zaanen J., Phys. Rev. Lett., 2008, 100, 166401; doi 10.1103/PhysRevLett.100.166401

12. Mishchenko A.S., Phys. Usp., 2009, 52, 1193; doi 10.3367/UFNe.0179.200912b.1259

13. Kastner M.A., Birgeneau R.J., Shirane G., Endoh Y., Rev. Mod. Phys., 1998, 70, 897; doi 10.1103/RevModPhys.70.897.

14. Lee P.A., Nagoasa N., Wen X.-G., Rev. Mod. Phys., 2006, 78, 17; doi 10.1103/RevModPhys.78.17

15. Lavrov A.N., Gandmakher V.F., Phys. Usp., 1998, 41, 223; doi 10.1070/PU1998v041n02ABEH000371

16. Komiya S., Ando Y., Sun X.F., Lavrov A.N., Phys. Rev. B, 2002, 65, 214535; doi 10.1103/PhysRevB.65.214535

17. Hussey H.E., Copper J.R., In: IRC Research Review, High Temperature Superconductivity, Liang W.Y. (Ed.), Cambridge University Press, Cambridge, 1998, 52.

18. Emin D., Polarons, Cambridge University Press, Cambridge, 2013.

19. Basov D.N., Timusk T., Rev. Mod. Phys., 2005, 77, 721; doi 10.1103/RevModPhys.77.721

20. Toyozawa Y., Physica B, 1983, 116, 7; doi 10.1016/0378-4363(83)90219-X

21. Lu J.P., Si Q., Phys. Rev. B, 1990, 42, 950; doi 10.1103/PhysRevB.42.950

22. Kittel Ch., Quantum Theory of Solids, Nauka, Moscow, 1967 (in Russian).

23. Emin D., Hillery M.S., Phys. Rev. B, 1989, 39, 6575; doi 10.1103/PhysRevB.39.6575

24. Weger M., Burlachkov I., Physica C, 1994, 235-240, 2387; doi 10.1016/0921-4534(94)92414-7

25. Baetzold R.C., Phys. Rev. B, 1990, 42, 56; doi 10.1103/PhysRevB.42.56

26. Markert J.T., Dalichaouch Y., Maple M.B., In: Physical Properties of High Temperature Superconductors I, Ginsberg D.M. (Ed.), Mir, Moscow, 1990, 265 (in Russian).

27. Mott N.F., Devis E.A., Electronic Processes in Non-Crystalline Materials, Mir, Moscow, 1974 (in Russian).

28. Appel J., In: Polarons, Firsov Yu.A. (Ed.), Nauka, Moscow, 1975, 11 (in Russian).

29. Verbist G., Peeters F.M., Devreese J.T., Phys. Scr., 1991, 39, 66; doi 10.1088/0031-8949/1991/T39/007

30. Adamowski J., Phys. Rev. B, 1989, 39, 3649; doi 10.1103/PhysRevB.39.3649

31. Verbist G., Peeters F.M., Devreese J.T., Phys. Rev. B, 1991, 43, 2712; doi 10.1103/PhysRevB.43.2712

32. Baimatov P.J., Khuzhakulov D.Ch., Sharipov Kh.T., Phys. Solid State, 1997, 39, 248; doi 10.1134/1.1129792.

33. Thomsen Ch., Cardona M., In: Physical properties of High Temperature Superconductors I, Ginsberg D.M. (Ed.), Mir, Moscow, 1990, 411 (in Russian).

34. Suprun S.G., Moizhes B.Ya., Fiz. Tverd. Tela, 1982, 24, 1571 (in Russian).

35. Hiramoto H., Toyozava Y., J. Phys. Soc. Jpn., 1985, 54, 245; doi 10.1143/JPSJ.54.245.

36. Vinetskii V.L., Meredov O., Yanchuk V.A., Teor. Eksp. Khim., 1989, 25, 641 (in Russian).

37. Tranquada J.M., Sternlieb B.J., Aye J.D., Nakamura Y., Uchida S., Nature, 1995, 375, 561; doi 10.1038/375561a0

38. Zaanen J., Osman O.Y., van Saarloos W., Phys. Rev. B, 1998, 58, R11868; doi 10.1103/PhysRevB.58.R11868

39. Varyukhin S.V., Zakharov A.A., Physica C, 1991, 185-189, 975; doi 10.1016/0921-4534(91)91711-C.

40. Ino A., Mizokawa T., Kobayashi K., Fujimori A., Sasagawa T., Kimura T., Kishio K., Tamasaku K., Eisaki H., Uchida S., Phys. Rev. Lett., 1998, 81, 2124; doi 10.1103/PhysRevLett.81.2124

41. Ino A., Kim C., Nakamura M., Yoshida T., Mizokawa T., Fujimori A., Shen Z.-X., Kakeshita T., Eisaki H., Uchida S., Phys. Rev. B, 2002, 65, 094504; doi 10.1103/PhysRevB.65.094504 


\section{Природа зовнішніх і внутрішніх самозахопних носіїв струму в слабкозлегованих купратних високотемпературних напівпровідниках}

\section{О.К. ГанєВ}

Фізичний факультет, Університет Узбекистану, 100174 Ташкент, Узбекистан

Теоретично вивчено природу зовнішніх і внутрішніх самозахопних носіїв струму в купратах. Енергії зв'язування і радіуси зовнішніх і внутрішніх великих поляронів і біполяронів в купратах обчислено варіаційно, використовуючи неперервну модель та адіабатичне наближення. Ми показали, що зовнішні і внутрішні тривимірні великі біполярони існують в слабозлегованих купратах, відповідно, при $\eta=\varepsilon_{\infty} / \varepsilon_{0}<0.127$ і $\eta<0.138$ [де $\varepsilon_{\infty}\left(\varepsilon_{0}\right)$ - оптична (статична) діелектрична стала].

Ключові слова: полярон, біполярон, самозахоплення, високотемпературні надпровідники 